\title{
A NOTE ON MULTIPLICATIVE COMMUTATORS OF DIVISION RINGS
}

\author{
ROOZBEH HAZRAT
}

\begin{abstract}
We give an example of a division ring $D$ whose multiplicative commutator subgroup does not generate $D$ as a vector space over its centre, thus disproving the conjecture posed in [1].
\end{abstract}

Let $D$ be a division ring. Denote by $F:=Z(D)$, the centre of $D$ and $D^{\prime}$ its multiplicative commutator subgroup, i.e., the group generated by the set of multiplicative commutators $\left\{x y x^{-1} y^{-1} \mid x, y \in D \backslash\{0\}\right\}$. There are classical results due to Herstein, Kaplansky and Scott, among others, showing that the group $D^{\prime}$ is "dense" in $D$ (see for example $[3, \S 13]$ ). In [1] the authors study the $F$-vector space $T(D)$ generated by the set of multiplicative commutators. They prove that if $T(D)$ is radical over $F$, then $D=F$, and if $\operatorname{dim}_{F} T(D)<\infty$, then $\operatorname{dim}_{F} D<\infty$. Furthermore, they prove that $T(D)$ contains all separable elements of $D$ and thus if $D$ is an algebraic division ring over its centre with $\operatorname{char}(D)=0$, then $T(D)=D$.

They then conjecture [1, Abstract and Conjecture 1] that a division ring is generated by all multiplicative commutators as a vector space over its centre, i.e., $T(D)=D$ for any arbitrary division ring.

Here we give a counterexample to this conjecture. In fact we show that the multiplicative subgroup $D^{\prime}$ can not recover $D$ as a vector space and $[D: T(D)]=\infty$.

We recall the Hilbert classical construction of division rings (see [2, §1]). Let $L$ be a field, $\sigma \in \operatorname{Aut}(L)$ and $F$ be the fixed field of $\sigma$. Let $D=L((x, \sigma))$ be the division ring of formal Laurent series, consisting of elements $\sum_{i=n}^{\infty} a_{i} t^{i}$, where $a_{i} \in L$ and $n \in \mathbb{Z}$, addition defined component-wise and multiplication by

$$
\left(\sum_{i=m}^{\infty} a_{i} t^{i}\right)\left(\sum_{j=n}^{\infty} b_{j} t^{j}\right)=\sum_{r=n+m}^{\infty}\left(\sum_{i+j=r} a_{i} \sigma^{i}\left(b_{j}\right)\right) t^{r} .
$$

By $[2, \S 1$, Lemma 4] if the order of $\sigma$ is infinite then $Z(D)=F$ and $[D: F]=\infty$ and if the order is a finite number $n$, then $Z(D)=F\left(\left(t^{n}\right)\right)$ and $[D: Z(D)]=n^{2}$. It is easy to see that $D=L((x, \sigma))$ is a valued division ring with value group $\mathbb{Z}$ as follows

$$
\begin{gathered}
v: D^{*} \longrightarrow \mathbb{Z}, \\
\sum_{i=n}^{\infty} a_{i} t^{i} \longmapsto n .
\end{gathered}
$$

Therefore we have

$$
D^{\prime} \subseteq \operatorname{ker}(v)=\left\{L+\sum_{i>0} a_{i} t^{i}\right\}
$$

Now if we choose an automorphism $\sigma$ of infinite order, since $Z(D)=F$, Equation 2 shows that $T(D) \subseteq$ $\left\{L+\sum_{i>0} a_{i} t^{i}\right\}$, and thus $[D: T(D)]=\infty$.

\section{REFERENCES}

[1] Aghabali, M., Akbari, S., Ariannejad, M., Madadi, A. Vector space generated by the multiplicative commutators of a division ring, J. Algebra Appl. 12 (2013), no. 8, 7 pp. 1

Key words and phrases. Division ring, Multiplicative commutator.

The author acknowledges Australian Research Council grant DP160101481. This work was done at the University of Münster, where the author was a Humboldt Fellow. 
[2] Draxl, P.K, Skew fields, London Mathematical Society Lecture Note Series, 81, Cambridge University Press, Cambridge, 1983. 1

[3] Lam, T.Y, A first course in noncommutative rings, volume 13, Graduate Texts in Mathematics. Springer-Verlag, New York, Second edition, 2001. 1

Western Sydney University, Australia

E-mail address: r.hazrat@westernsydney.edu.au 